\title{
Optimal Solution of Neutrosophic Linear Fractional Programming Problems With Mixed Constraints
}

SAPAN DAS ( $\square$ cool.sapankumar@gmail.com )

National Institute of Technology Jamshedpur https://orcid.org/0000-0002-6716-4427

S A Edalatpanah

Ayandegan Institute of Higher Education

\section{Research Article}

Keywords: Neutrosophic linear fractional programming, Crisp linear fractional programming, Triangular neutrosophic numbers, Ranking function

Posted Date: January 7th, 2022

DOI: https://doi.org/10.21203/rs.3.rs-1225608/v1

License: (1) This work is licensed under a Creative Commons Attribution 4.0 International License.

Read Full License 
Optimal solution of Neutrosophic Linear Fractional Programming Problems with mixed constraints

Sapan Kumar Das ${ }^{1^{*}}$, S. A. Edalatpanah ${ }^{2}$,

1. Ministry of Finance, Department of Revenue, Govt. of India Email-cool.sapankumar@gmail.com

2. Department of Applied Mathematics, Ayandegan Institute of Higher Education, Tonekabon, Iran

\begin{abstract}
:
In this paper, Linear Fractional Programming (LFP) problems have been extended to neutrosophic sets (NSs) and the operations and functionality of these laws are studied. Moreover, the new algorithm is based on aggregation ranking function and arithmetic operations of triangular neutrosophic sets (TNSs). Furthermore, for the first time, in this paper, we take up a problem where the constraints are both equality and inequality neutrosophic triangular fuzzy number. Lead from genuine issue, a few numerical models are considered to survey the legitimacy, profitability and materialness of our technique. At last, some numerical trials alongside one contextual analysis are given to show the novel techniques are better than the current strategies.
\end{abstract}

Keywords: Neutrosophic linear fractional programming, Crisp linear fractional programming, Triangular neutrosophic numbers, Ranking function.

\title{
1. Introduction
}

These days, the issue of LFP is one of the most key tool feasible analyses. Various physical issues can be converted into a LFP model; see $[10,22]$. As a result the present template is the crucial for the present applications in various genuine zones, for example, creation arranging, money related area, medicinal services and all designing fields [10]. Be that as it may, in certifiable applications, conviction, unwavering quality and accuracy of information is regularly deceptive.In general the ultimate goal of LFP just relies upon a set number of imperatives; consequently a significant part of the gathered data has little effect on the arrangement.It is helpful to think about the information on specialists regarding the parameters as invisible information. Zadeh proposed fuzzy theory [5], researchers used it when the data are imprecise. Numerous specialists have deciphered numerous models of fuzzy linear programming and fuzzy linear fractional programming problem. They have taken different approaches to take care of the 
fuzzy issue like (i) where coefficients are fuzzy, (ii) variables are fuzzy, (iii) both are fuzzy. In the event that the issue consider both fuzzy, at that point it is called completely fuzzy programming issue. That kind of issue was planned by Zimmerman. After that Edalatpanah [24], Das [20], Das et al. [11] has defined to tackle FFLP issue with the assistance of ranking function and lexicographic technique.Nayak\&Ojha [39-40]have proposed a technique to take care of multi-objective LFP issue with interval parameters. Some of analysts [12-15,23,35-38,41]have proposed various strategies for tackling multi-objective FLFP issue. Veeramani and Sumathi[4]proposed a technique for tackling FFLFP issue by utilizing the ideas of $\alpha, r$ acceptable optimal value of a LFP problem with fuzzycoefficients and fuzzy decision variables and build up a strategy to register them. As of late, Das et al.[10] have proposed a strategy for tackling fuzzy LFP issue by utilizing multi-target LP issue. These creators utilized the strategy for lexicographic procedure and the FFLFP issue convert into multi-target LP issue; see additionally [10, 11-19,25].

Afterward, intuitionistic fuzzy hypothesis was presented by Atanassov [3], some mathematician likewise utilized it to display vulnerability in streamlining issues. In any case, these two ideas can just explore inadequate data, not wide data.Be that as it may, Smarandache [27] tackled this issue by adding an autonomous indeterminacy enrollment to intuitionistic fuzzy sets and this strategy is called neutrosophic set. Due to their greatness attribution the neutrosophic issue is becoming exceptionally quick. As a matter of first importance, Ye [54-55] proposed a capacity including neurosophic numbers and utilized it for taking care of neutrosophic linear programming problem. Presently numerous specialists consider the neutrosophic capacities to take care of different issues like goal programming [43-47,50-52], non-linear programming [13,48], geometric programming [52], integer programming [47] and data envelopment analysis [32]. Abdel Basset and Mohamed [28] proposed a neutrosophic triangular numbers to take care of LFP issues. They consider neutrosophic LFP (NLFP) issue into multi-objective LFP issue and afterward convert to single target LFP issue. By utilizing existing technique [28] to acquire arrangements are not accurate and the requirements are taken disparity. It is hard to utilize the current technique [28] for taking care of FFLFP issue with both equity and imbalance limitations. Recently, Das and Dash [53] consider a neurtorsophic linear fractional 
programming problem with mixed constraints with using score function. To overcome this existing method [53], the aim of this paper is introduced aggregation ranking function.

To conquer these weaknesses, another technique is proposed for discovering NLFP issue with both uniformity and imbalance limitations. This examination, at the first occasion when we use total positioning capacity to tackle neutrosophic LFP issue and it will change over to fresh LFP issue. This fresh LFP issue is explained by any standard techniques.

The remainder of the paper is composed by in this way: some essential ideas and documentations are available in clause 2 . In clause 3, the general type of direct partial programming (LFP) issue is introduced. The general type of neutosophic LFP issue is displayed in clause 4.In clause 5, another methodology is introduced for taking care of NLFP issues with imbalance requirements and fairness imperatives having unhindered neutrosophic triangular numbers are introduced. To outline the utilization of the proposed technique for a mechanical application is understood in clause 6 . At long last, end has been attracted the last segment.

\section{Preliminaries}

An audit of significant ideas and meanings of neutrosophic set is introduced right now.

Definition 1. [27]

Assume $X$ stick a universal neutosophic set in addition to $s \in X$. A neutrosophic set $X$ may be defined viathree membership functions for truth, indeterminacy along with falsity and denoted by $\tau_{M}(s), \rho_{M}(s)$ and $v_{M}(s)$. These are realistic abnormal subgroups containing $] 0^{-}, 1^{+}[$. There may be no limitation on the whole of $\tau_{M}(s), \rho_{M}(s)$ and $v_{M}(s)$,so $0^{-} \leq \sup \tau_{M}(s)+\sup \rho_{M}(s)+\sup v_{M}(s) \leq 3^{+}$.

\section{Definition 2. [29]}

A single-valued neutrosophic set (SVNS)M excess of $X$ may be an article giving powerful structure $M=\left\{s, \tau_{M}(s), \rho_{M}(s), v_{M}(s)\right\}$, where $\mathrm{X} \quad$ be a space of discourse, $\tau_{M}(s): X \rightarrow[0,1], \rho_{M}(s): X \rightarrow[0,1]$ and $v_{M}(s): X \rightarrow[0,1] \quad$ with $\quad 0<\tau_{M}(s)+\rho_{M}(s)+v_{M}(s)<3$ $0 \leq T A(x)+I A(x)+F A(x) \leq 3$ for all $s \in X$.

Definition 3 [28]. A triangular neutrosophic number (TNNs) is signifiedvia $M=<\left(a^{q}, a^{r}, a^{s}\right),(\mu, i, \omega)>$ is an extended version of the three membership functions for the truth, indeterminacy, and falsity of $\mathrm{s}$ can be defined as follows: 


$$
\begin{gathered}
\tau_{M}(s)=\left\{\begin{array}{lc}
\frac{\left(s-a^{q}\right)}{\left(a^{r}-a^{q}\right)} \mu & a^{q} \leq s<a^{r}, \\
\mu & \mathrm{s}=a^{q}, \\
\frac{\left(a^{s}-s\right)}{\left(a^{s}-a^{r}\right)} \mu & a^{r} \leq s<a^{s}, \\
0 & \text { something else. }
\end{array}\right. \\
\rho_{M}(s)=\left\{\begin{array}{lc}
\frac{\left(a^{r}-s\right)}{\left(a^{r}-a^{s}\right)} i, & a^{q} \leq s<a^{r}, \\
i, & \mathrm{~s}=a^{r}, \\
\frac{\left(s-a^{s}\right)}{\left(a^{s}-a^{u}\right)} i, & a^{r} \leq s<a^{s}, \\
1, & \text { something else. }
\end{array}\right. \\
v_{M}(s)= \begin{cases}\frac{\left(a^{m}-s\right)}{\left(a^{m}-a^{l}\right)} \omega, & a^{q} \leq s<a^{r}, \\
\frac{\left(s-a^{s}\right)}{\left(a^{s}-a^{r}\right)} \omega, & a^{r} \leq s<a^{s}, \\
1, & \text { something else. }\end{cases}
\end{gathered}
$$

Where, $\mathrm{O} \leq \tau_{M}(s)+\rho_{M}(s)+v_{M}(s) \leq 3, s \in M$. Additionally, when $a^{q} \geq 0, M$ is called anonnegative TNN. Similarly, when $a^{q}<0, M$ becomes a negative TNN.

\section{Definition 4 [28]. Arithmetic Operation}

Suppose $A_{1}{ }^{M}=<\left(a^{q}, b^{r}, c^{s}{ }_{1}\right),\left(\mu_{1}, v_{1}, \omega_{1}\right)>$ and $A_{2}{ }^{M}=<\left(a^{q}{ }_{2}, b^{r}{ }_{2}, c^{s}{ }_{2}\right),\left(\mu_{2}, v_{2}, \omega_{2}\right)>$ be two TNNs. Then the arithmetic relations are defined as:

(i) $A_{1}^{M} \oplus A_{2}^{M}=<\left(a^{q}{ }_{1}+a^{q}{ }_{2}, b_{1}^{r}+b_{2}^{r}, c_{1}^{s}+c^{s}{ }_{2}\right),\left(\mu_{1} \wedge \mu_{2}, v_{1} \vee v_{2}, \omega_{1} \vee \omega_{2}\right)>$

(ii) $A_{1}^{M}-A_{2}^{M}=<\left(a^{q}{ }_{1}-c_{2}^{s}, b^{r}{ }_{1}-b^{r}{ }_{2}, c^{s}{ }_{1}-a^{q}{ }_{2}\right),\left(\mu_{1} \wedge \mu_{2}, v_{1} \vee v_{2}, \omega_{1} \vee \omega_{2}\right)>$

(iii) $A_{1}{ }^{M} \otimes A_{2}{ }^{M}=<\left(a^{q}{ }_{1}^{q} a_{2}, b_{1}^{r} b_{2}^{r}, c_{1}^{s} c^{s}{ }_{2}\right),\left(\mu_{1} \wedge \mu_{2}, v_{1} \vee v_{2}, \omega_{1} \vee \omega_{2}\right)>$, if $a_{1}^{q}>0, b_{1}^{r}>0$,

(iv) $\lambda A_{1}^{M}= \begin{cases}\left\langle\left(\lambda a^{q}, \lambda b_{1}^{r}, \lambda c_{1}^{s}\right),\left(\mu_{1}, v_{1}, \omega_{1}\right)\right\rangle, & \text { if } \lambda>0 \\ \left\langle\left(\lambda c^{s}{ }_{1}, \lambda b^{r}, \lambda a_{1}^{q}\right),\left(\mu_{1}, v_{1}, \omega_{1}\right)\right\rangle, & \text { if } \lambda<0\end{cases}$ 


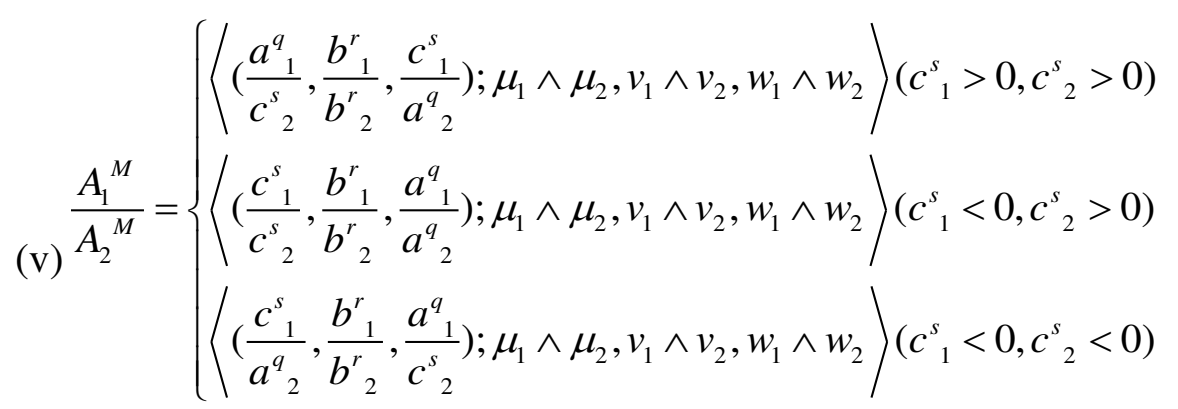

Definition 5 [10]. Arithmetic Operation

Assume $A^{M}$ and $B^{M}$ be two TNNs. Then the ranking function is:

(i) $\quad A^{M} \leq B^{M}$ if and only if $\mathfrak{R}\left(A^{M}\right) \leq \mathfrak{R}\left(B^{M}\right)$.

(ii) $\quad A^{M}<B^{M}$ if and only if $\mathfrak{R}\left(A^{M}\right)<\mathfrak{R}\left(B^{M}\right)$.

Where $\mathfrak{R}($.$) is a ranking function.$

Here, we present a new type of ranking function:

Definition 6. One can compare any two TNNs based on the ranking functions. Let $A^{N}=<(a, b, c) ;(\mu, v, w)>$ be a TNN: then $\mathfrak{R}\left(A^{N}\right)=\frac{a+b+c}{9}(\mu+(1-v)+(1-w))$.

Example-1. Let $A^{N}=((4,8,10) ; 0.5,0.3,0.6)$ then $\mathfrak{R}\left(A^{N}\right)=3.91$

Since $\mathfrak{R}(\tilde{a}+\tilde{b}) \neq \mathfrak{R}(\tilde{a})+\mathfrak{R}(\tilde{b})$, therefore, we define an aggregation ranking function as follows:

Definition 7. Let $A_{i}^{N}=<\left(a_{i}, b_{i}, c_{i}\right) ;\left(\mu_{i}, v_{i}, w_{i}\right)>$ be some TNNs: then the aggregation ranking function is as follows:

$\mathfrak{R}\left(\sum_{i=1}^{n} A_{i}^{N}\right)=\frac{\left(2+\min \mu_{i}-\max v_{i}-\max w_{i}\right)}{9} \sum_{i=1}^{n}\left(a_{i}+b_{i}+c_{i}\right)$

Example-2. Let $A^{N}=((4,8,10) ; 0.5,0.3,0.6)$ and $B^{N}=((3,7,11) ; 0.4,0.5,0.6)$ then based on Definition 6 we get:

$\mathfrak{R}\left(A^{N}\right)=3.91$,

$\mathfrak{R}\left(A^{N}\right)=3.03$.

But, by Definition 4: 
$A^{N}+B^{N}=((7,15,21) ; 0.4,0.5,0.6)$

$\mathfrak{R}\left(A^{N}+B^{N}\right)=6.21 \neq \mathfrak{R}\left(A^{N}\right)+\mathfrak{R}\left(B^{N}\right)=6.94$.

However, based on Definition 7. We get,

$\mathfrak{R}\left(A^{N}+B^{N}\right)=\frac{(2+0.4-0.5-0.6)}{9} \times(22+21)=6.94=\mathfrak{R}\left(A^{N}\right)+\mathfrak{R}\left(B^{N}\right)$.

\section{Linear fractional programming model}

Right now, general model of LFP issue is examined. Besides, Charnes plus Cooper's [1] linear transformation is outlined.

$$
\operatorname{Max} \mathrm{Z}(\mathrm{s})=\frac{\sum c_{j}^{t} s_{j}+p}{\sum d_{j}^{t} s_{j}+q}=\frac{F(s)}{G(s)}
$$

Subject to

$$
s \in S=\left\{s \in R^{n} . A s \leq b, s \geq 0\right\}
$$

where $\mathrm{j}=1,2, \ldots . ., \mathrm{n}, \quad A \in R^{m \times n}, b \in R^{m}, c, d \in R^{n}, p, q \in R$. Just for values of $\mathrm{s}, \mathrm{G}$ (s) might be equal to zero. In order to keep away from given situations, one requires which either $\{s \geq 0, A s \leq b, \Rightarrow G(s)>0\}$ or $\{s \geq 0, A s \leq b, \Rightarrow G(s)<0\}$. Since appliance, put on a well-known LFP problem provides the status that

$\{s \geq 0, A s \leq b, \Rightarrow G(s)>0\}$

Theorem 3.1[3]: Accept that no point $(s, 0)$ as well as $s \geq 0$ is feasible in the accompanying linear programming issue.

$$
\begin{gathered}
\text { Max } c^{t} s+p t \\
\text { Subject to } \begin{array}{c}
d^{t} s+q t=1, \\
A s-b t=0, \\
t>0, s \geq 0, s \in R^{n}, t \in R .
\end{array}
\end{gathered}
$$

At that point, with the state of connection (2), the LFP issue (1) is equal in order to generallinear programming issue model (3).

Presently, think about the two associated issues

$$
\text { Max } t F(s / t)
$$

Subject to 


$$
\begin{aligned}
& A(s / t)-b \leq 0, \\
& t G(s / t)=1, \\
& t>0, s \geq 0 .
\end{aligned}
$$

and,

$$
\operatorname{Max} t F(s / t)
$$

Subject to

$$
\begin{aligned}
& A(s / t)-b \leq 0, \\
& t G(s / t) \leq 1, \\
& t>0, s \geq 0 .
\end{aligned}
$$

where, model (4) is acquired from model (1) through the change $t=1 / G(s), z=t s$ and model (5) contrasts from the model (4) by supplanting the equity constraints $t G(s / t)=1$ by inequality requirements $t G(s / t) \leq 1$.

Theorem 3.2[3]: On the off chance that the model (1) may be well-knownconcave-convex programming issue that arrives at a most extreme at a point $S^{*}$, then the comparing changed issue model (5)rises up sensational same limit at a point $\left(t^{*}, s^{*}\right)$ wherever $\frac{s^{*}}{t^{*}}=x^{*}$. Additionally model (5) seems to have concave objective function along with a feasible convex set.

Suppose that:

$$
\begin{aligned}
& \operatorname{Max} Z(s)=\frac{F(s)}{G(s)} \\
& \text { Subject to } s \in S=\left\{s \in R^{n} . A s \leq b, s \geq 0\right\},
\end{aligned}
$$

Where $F(s)$ may be concave as well as negative for every $s \in S$ and $G(s)$ may be concave as well as positive as to $\mathrm{S}$, then

$$
\operatorname{Max}_{s \in S} \frac{F(s)}{G(s)} \Leftrightarrow \operatorname{Min}_{s \in S} \frac{-F(s)}{G(s)} \Leftrightarrow \operatorname{Max}_{s \in S} \frac{G(s)}{-F(s)}
$$

where $-F(s)$ are often convex plus positive. In this manner, the issue (6) may be changed over into a well-knownconcave-convex programming issue changed into the accompanying linear programming issue: 


\section{$\operatorname{Max} t F(s / t)$}

Subject to

$$
\begin{aligned}
& A(s / t)-b \leq 0, \\
& t G(s / t) \leq 1, \\
& t>0, s \geq 0 .
\end{aligned}
$$

\section{Neutorsophic LFP model dependent on triangular numbers}

At the present time, we proposed a general model for NLFP issue is examined.

$$
\operatorname{Max}\left(\text { or Min) } Z\left(s^{M}\right)=\frac{\sum c_{j}^{M} s_{j}+p^{M}}{\sum d_{j}^{M} s_{j}+q^{M}}\right.
$$

Subject to

$$
\begin{gathered}
\sum a_{i j}^{M} s_{j} \leq b_{i}^{M}, i=1,2, \ldots, m, \\
\sum a_{i j}^{M} s_{j}=1^{M}, i=1,2, \ldots ., m, \\
s_{j} \geq 0, j=1,2, \ldots, n .
\end{gathered}
$$

The first run through, Abdel-Basset [28] proposed another strategy for taking care of NLFP issue by utilizing a multi-target LP issue with just inequality constraints and gets an ideal arrangement. They utilize a technique to change over to its multi-objective, which is increment the quantity of factors and imperatives, while in our strategy, the quantity of requirements and factors stays unaltered. The proposed model contrasted with other technique is very tedious and expensive.

\section{Proposed strategy}

Right now, here we solve the model (8), we propose the following algorithm:

Step 1. Construct the problem as the model (8).

Step 2. By Definition 5, We assume that $c_{j}^{N}, p^{N}, d_{j}^{N}, q^{N}, a_{i j}^{N}$ and $b_{i}^{N}$ are triangular neutrosophic for each $i=1,2, \ldots, m \& i=1,2, \ldots ., n$ therefore, the model (8) can be composed as :

$\operatorname{Max}($ or Min $) Z\left(s^{M}\right)=\frac{\sum\left(c_{j 1}, c_{j 2}, c_{j 3} ; \mu_{c}, v_{c}, w_{c}\right) s_{j}+\left(p_{1}, p_{2}, p_{3} ; \mu_{p}, v_{p}, w_{p}\right)}{\sum\left(d_{j 1}, d_{j 2}, d_{j 3} ; \mu_{d}, v_{d}, w_{d}\right) s_{j}+\left(q_{1}, q_{2}, q_{3} ; \mu_{q}, v_{q}, w_{q}\right)}$

subject to

$$
\begin{aligned}
& \sum\left(a_{i j 1}, a_{i j 2}, a_{i j 3} ; \mu_{a}, v_{a}, w_{a}\right) s_{j} \leq\left(b_{i 1}, b_{i 2}, b_{i 3} ; \mu_{b}, v_{b}, w_{b}\right), i=1,2, \ldots ., m . \\
& \sum\left(a_{i j 1}, a_{i j 2}, a_{i j 3} ; \mu_{a}, v_{a}, w_{a}\right) s_{j}=1^{M}, i=1,2, \ldots ., m .
\end{aligned}
$$




$$
s_{j} \geq 0, j=1,2, \ldots, n .
$$

Step 3. Utilizing aggregation ranking function, the model (9) convert into model (10) as : $\operatorname{Max}($ or Min $) Z\left(s^{M}\right)=\frac{\mathfrak{R} \sum\left(c_{j 1}, c_{j 2}, c_{j 3} ; \mu_{c}, v_{c}, w_{c}\right) s_{j}+\left(p_{1}, p_{2}, p_{3} ; \mu_{p}, v_{p}, w_{p}\right)}{\Re \sum\left(d_{j 1}, d_{j 2}, d_{j 3} ; \mu_{d}, v_{d}, w_{d}\right) s_{j}+\left(q_{1}, q_{2}, q_{3} ; \mu_{q}, v_{q}, w_{q}\right)}$ subject to

$$
\begin{gathered}
\Re \sum\left(a_{i j 1}, a_{i j 2}, a_{i j 3} ; \mu_{a}, v_{a}, w_{a}\right) s_{j} \leq \mathfrak{R}\left(b_{i 1}, b_{i 2}, b_{i 3} ; \mu_{b}, v_{b}, w_{b}\right), i=1,2, \ldots ., m . \\
\mathfrak{R} \sum\left(a_{i j 1}, a_{i j 2}, a_{i j 3} ; \mu_{a}, v_{a}, w_{a}\right) s_{j}=\mathfrak{R}\left(1^{M}\right), i=1,2, \ldots ., m . \\
s_{j} \geq 0, j=1,2, \ldots, n .
\end{gathered}
$$

Step 4. Utilizing Definition 7, the model (10) is changed over into next crisp model:

$$
\begin{aligned}
& {\left[\frac{\left(2+\min \mu_{c}-\max v_{c}-\max w_{c}\right)}{9} \sum_{j=1}^{n}\left(c_{j 1}+c_{j 2}+c_{j 3}\right)\right] s_{j}+} \\
\operatorname{Max}(\text { or Min }) Z\left(s^{M}\right)= & {\left[\frac{\left(2+\min \mu_{p}-\max v_{p}-\max w_{p}\right)}{9} \sum_{j=1}^{n}\left(p_{1}+p_{2}+p_{3}\right)\right] } \\
& {\left[\frac{\left(2+\min \mu_{d}-\max v_{d}-\max w_{d}\right)}{9} \sum_{j=1}^{n}\left(d_{j 1}+d_{j 2}+d_{j 3}\right)\right] s_{j}+} \\
& {\left[\frac{\left(2+\min \mu_{q}-\max v_{q}-\max w_{q}\right)}{9} \sum_{j=1}^{n}\left(q_{1}+q_{2}+q_{3}\right)\right] }
\end{aligned}
$$

subject to

$$
\begin{gathered}
{\left[\frac{\left(2+\min \mu_{a}-\max v_{a}-\max w_{a}\right)}{9} \sum_{j=1}^{n}\left(a_{i j 1}+a_{i j 2}+a_{i j 3}\right)\right] s_{j} \leq\left[\frac{\left(2+\min \mu_{b}-\max v_{b}-\max w_{b}\right)}{9} \sum_{j=1}^{n}\left(b_{i 1}+b_{i 2}+b_{i 3}\right)\right]} \\
{\left[\frac{\left(2+\min \mu_{a}-\max v_{a}-\max w_{a}\right)}{9} \sum_{j=1}^{n}\left(a_{i j 1}+a_{i j 2}+a_{i j 3}\right)\right] s_{j}=1,} \\
s_{j} \geq 0, j=1,2, \ldots, n .
\end{gathered}
$$

Step 5. The model (11) is presently a crisp LFP issue. Presently the Model (11) is transformedinto LP issue by utilizing the Charnes-Cooper strategy (or any well known technique). 
Step 6. From Step 5, we use LINGO to take care of the fresh LP issue and get the ideal arrangement.

The flow chart describes the procedure of the proposed method as shown in Fig. 1.

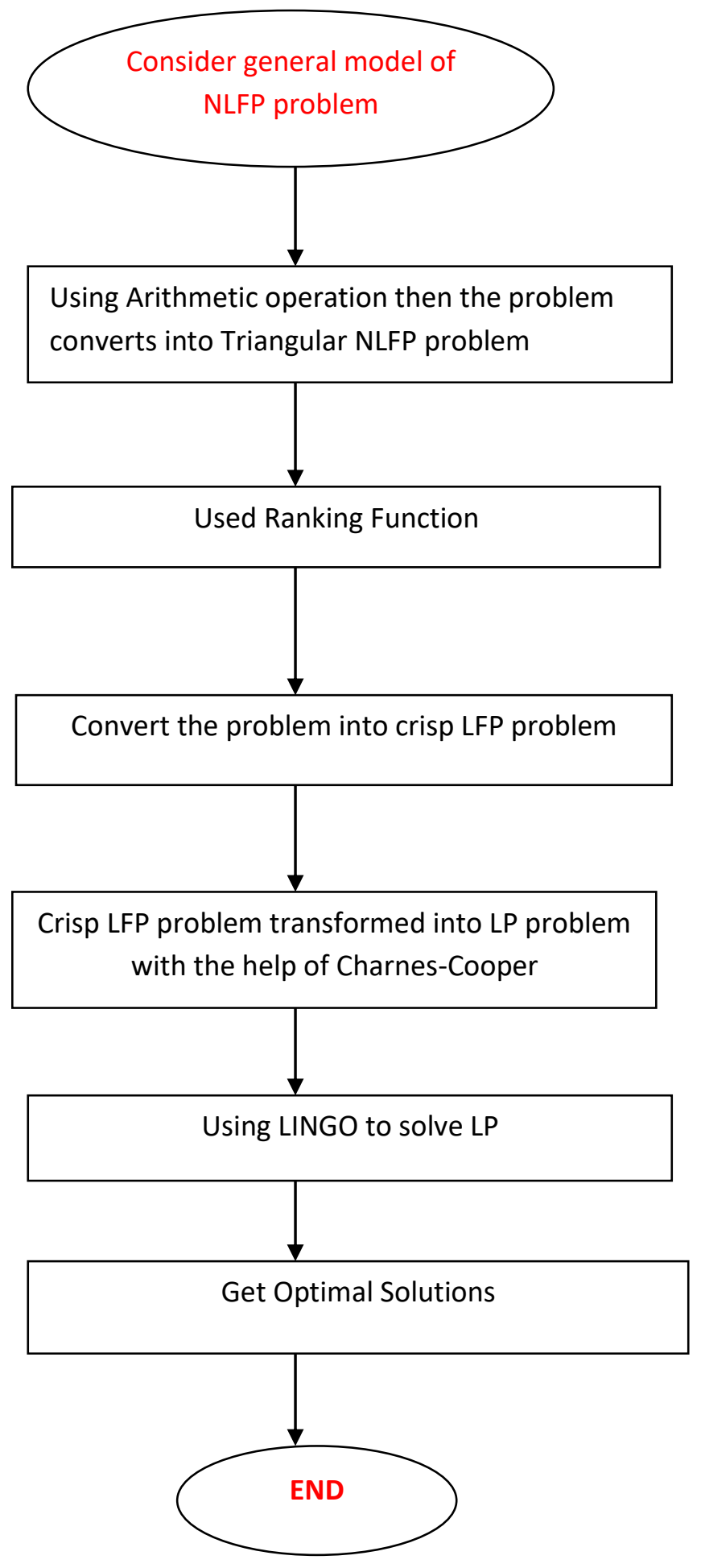




\section{Numerical Example}

Here, we select a case of [28] to represent the model alongside correlation of existing technique.

\section{Example-1 (Industrial Application)}

Organization fabricates three sorts of items $\mathrm{A}, \mathrm{B}$ and $\mathrm{C}$ with benefit round 8,7 and9 dollars for every component, separately. Be that as it may, the expense for every one component of the items isround 8,9 and 6 dollars, individually. Additionally, it is accepted that a fixed expense of round1.5 dollars is added to the cost work because of anticipated term through the procedure ofproduction. Assume the materials required for assembling the items A, B and C areabout 4, 3 and 5component for each pound, separately. The stockpile given crude materialis confined to round 28 pounds. Worker hour's accessibility for item A is around five hours, for item $\mathrm{B}$ is around three hours, and that for $\mathrm{C}$ is around three hours in manufacturingper component. Absolute worker hour's accessibility is round 20 hours day by day. Decide how many products of $\mathrm{A}, \mathrm{B}$ and $\mathrm{C}$ ought to be made so as to maximize the complete benefit. Alsoduring the entire procedure, the administrator wavers in expectation of parametric qualities due tosome wild factors.

Let $x_{1}, x_{2}$ and $x_{3}$ component be the measure of $\mathrm{A}, \mathrm{B}$ and $\mathrm{C}$, individually to be created. Afterprediction of evaluated parameters, the above issue can be defined as the followingNLFPP:

\section{Step-1}

$$
\operatorname{Max} Z=\frac{8^{N} x_{1}+7^{N} x_{2}+9^{N} x_{3}}{8^{N} x_{1}+9^{N} x_{2}+6^{N} x_{3}+1.5^{N}}
$$

Subject to

$$
\begin{gathered}
4^{N} x_{1}+3^{N} x_{2}+5^{N} x_{3} \leq 28^{N} \\
5^{N} x_{1}+3^{N} x_{2}+3^{N} x_{3} \leq 20^{N} \\
x_{1}, x_{2}, x_{3} \geq 0 .
\end{gathered}
$$

Here we consider, 


$$
\begin{aligned}
& 8^{N}=(7,8,9 ; 0.5,0.8,0.3), 7^{N}=(6,7,8 ; 0.2,0.6,0.5), 9^{N}=(8,9,10.8,0.1,0.4), 6^{N}=(4,6,8 ; 0.75,0.25,0.1), \\
& 1.5^{N}=(1,1.5,2 ; 0.75,0.5,0.25), 4^{N}=(3,4,5 ; 0.4,0.6,0.5), 3^{N}=(2,3,4 ; 1,0.25,0.3), 5^{N}=(4,5,6 ; 0.3,0.4,0.8) \\
& 28^{N}=(25,28,30 ; 0.4,0.25,0.6), 20^{N}=(18,20,22 ; 0.9,0.2,0.6)
\end{aligned}
$$

\section{Step-2}

Presently the issue is modified as follows:

$\operatorname{Max} Z=\frac{(7,8,9 ; 0.5,0.8,0.3) x_{1}+(6,7,8 ; 0.2,0.6,0.5) x_{2}+(8,9,10 ; 0.8,0.1,0.4) x_{3}}{(7,8,9 ; 0.5,0.8,0.3) x_{1}+(8,9,10.8,0.1,0.4) x_{2}+(4,6,8 ; 0.75,0.25,0.1) x_{3}+(1,1.5,2 ; 0.75,0.5,0.25)}$

Subject to

$$
\begin{gathered}
(3,4,5 ; 0.4,0.6,0.5) x_{1}+(2,3,4 ; 1,0.25,0.3) x_{2}+(4,5,6 ; 0.3,0.4,0.8) x_{3} \leq(25,28,30 ; 0.4,0.25,0.6) \\
(4,5,6 ; 0.3,0.4,0.8) x_{1}+(2,3,4 ; 1,0.25,0.3) x_{2}+(2,3,4 ; 1,0.25,0.3) x_{3} \leq(18,20,22 ; 0.9,0.2,0.6) \\
x_{1}, x_{2}, x_{3} \geq 0
\end{gathered}
$$

\section{Step-3}

Utilizing Step 3 the aggregation function the issue might be composed as follows:

$\operatorname{Max} Z=\frac{\mathfrak{R}\left[(7,8,9 ; 0.5,0.8,0.3) x_{1}+(6,7,8 ; 0.2,0.6,0.5) x_{2}+(8,9,10 ; 0.8,0.1,0.4) x_{3}\right]}{\mathfrak{R}\left[(7,8,9 ; 0.5,0.8,0.3) x_{1}+(8,9,10.8,0.1,0.4) x_{2}+(4,6,8 ; 0.75,0.25,0.1) x_{3}+(1,1.5,2 ; 0.75,0.5,0.25)\right]}$

\section{Subject to}

$\mathfrak{R}\left[(3,4,5 ; 0.4,0.6,0.5) x_{1}+(2,3,4 ; 1,0.25,0.3) x_{2}+(4,5,6 ; 0.3,0.4,0.8) x_{3} \leq(25,28,30 ; 0.4,0.25,0.6)\right]$

$\mathfrak{R}\left[(4,5,6 ; 0.3,0.4,0.8) x_{1}+(2,3,4 ; 1,0.25,0.3) x_{2}+(2,3,4 ; 1,0.25,0.3) x_{3} \leq(18,20,22 ; 0.9,0.2,0.6)\right]$

$$
x_{1}, x_{2}, x_{3} \geq 0
$$

\section{Step-4}

By applying Step 4. And the problem converted into the crisp model.

$$
\operatorname{Max} Z=\frac{3.73 x_{1}+2.1 x_{2}+6.9 x_{3}}{3.73 x_{1}+6.9 x_{2}+4.8 x_{3}+1}
$$


Subject to

$$
\begin{gathered}
1.73 x_{1}+2.45 x_{2}+1.83 x_{3} \leq 14.29 \\
1.83 x_{1}+2.45 x_{2}+2.45 x_{3} \leq 14 \\
x_{1}, x_{2}, x_{3} \geq 0 .
\end{gathered}
$$

\section{Step-5}

As per the Step-5, we transform the crisp LFP problem into the crisp LP problem and solved by any popular method.

$$
\operatorname{Max} Z(y, t)=3.73 y_{1}+2.1 y_{2}+6.9 y_{3}
$$

Subject to

$$
\begin{gathered}
3.73 y_{1}+6.9 y_{2}+4.8 y_{3}+t \leq 1, \\
1.73 y_{1}+2.45 y_{2}+1.8 y_{3}-14.29 t \leq 0, \\
1.83 y_{1}+2.45 y_{2}+2.45 y_{3}-14 t \leq 0, \\
y_{1}, y_{2}, y_{3}, t \geq 0 .
\end{gathered}
$$

\section{Step-6}

The ideal arrangement of the above CLP issue is explained by LINGO 18.0 and gets the ideal arrangement is:

$$
y_{1}=0=y_{2}, y_{3}=0.2, t=0.25
$$

Finally, the objective solution is 1.4 .

Table-1. Comparison of the objective function

\begin{tabular}{|c|c|c|}
\hline Objective value & Proposed Method & Existing Method[28] \\
\hline$y_{1}$ & 0 & 0 \\
\hline$y_{2}$ & 0 & 0 \\
\hline$y_{3}$ & 0.2 & 0.0893 \\
\hline $\mathrm{T}$ & 0.25 & 0.1429 \\
\hline $\mathrm{Z}$ & 1.4 & 1 \\
\hline
\end{tabular}


In Abdel Basset et al.'s method [28] the optimal solution of the objective functions are:

$$
\tilde{y}_{1}=\left(y_{1}, y_{2}, y_{3}\right)=(0,0,0.0893), T=0.1429
$$

The objective value is:

$$
\mathrm{Z}=1
$$

In our proposed the optimal solution of the objective functions is:

$$
\tilde{y}_{1}=\left(y_{1}, y_{2}, y_{3}\right)=(0,0,0.2), T=0.25
$$

The objective value is:

$$
\mathrm{Z}=1.4
$$

By comparing the results of proposed method with Abdel-Basset et al.'s method, based on Definition 2.7, we can conclude that our result is better than the existing result, because:

$$
\left(\tilde{c} \tilde{x}^{*}\right) \text { proposed method }=1.4>\left(c^{t} x\right) \text { Abdel-Basset et al.'s method }=1
$$

From the above table, unmistakably the proposed target work is expanding contrast with existing strategy [28]. Therefore, our method is superior and the elapsed time is very less than the existing method. Subsequently we may reason that the proposed arrangement is increasingly exact.

It is pointed out that the proposed method is no restriction of all variables and parameters and the obtained results are satisfied all the constraints. We used in neutrosophic sets of the objective function is neglected by linear ranking function in decision makers.

Our model represents reality efficiently than existing model, because we consider all aspects of decision-making process in our calculations (i.e. the truthiness, indeterminacy, and falsity degree). Our model reduces complexity of problem, by reducing the number of constraints and variables. Their model is a time-consuming and complex, but our model is not.

In our proposed model, we solved both inequality and equality constraints (mixed constraints), but in existing model they have concentrated only inequality constraints. Hence, it is very easy and comfortable for applied in real life application as compared as to existing methods.

\section{Conclusions}

Right now, present the neutrosophic LFP and propose a novel model to illuminate it. Another ranking function is acquainted with defeat the current strategy. The new ranking function gives the augmenting the target work an incentive in neutrosophic LFP issue. Additionally, we utilize a 
mechanical application issue to represent the common sense and legitimacy of the proposed technique. At long last, from the acquired outcomes, it very well may be inferred that the model is proficient and helpful.

\section{Compliance with Ethical Standards}

\subsection{Conflict of Interest}

All the authors declare that they have no conflict of interest.

8.2 Ethical approval

This article does not contain any studies with human participants or animals performed by any of the authors.

\section{References i}

[1]iA.iCharnes iand iW.iW.iCooper, iProgramming iwith ilinear ifractional ifunctions, iNav.iRes.iLog.iQuar.i9 i(1962) i181-186.

[2] iB.iPop iand iI.iM. iStancu-Minasian, iA imethod iof isolving ifully ifuzzified ilinear ifractional iprogramming iproblems, iJ.iof iAppl.iMath.iComp.i27 i(2008) i227-242.

[3]iKrassimirT.Atanassov, iIntuitionistic ifuzzy isets, iFuzzy isets, i20(1986) i87-96.

[4]iC.iVeeramani iand iM.iSumathi, iFuzzy imathematical iprogramming iapproach ifor isolving ifuzzy ilinear ifractional iprogramming iproblem, iRAIRO iOper.iRes.i48 i(2014) i109122.

[5]iL.iA.iZadeh, iFuzzy iSets, inf.and iCont.i8 i(1965) i338-353.

[6]iN.iMahdavi- iAmiri, iS.iH.iNasseri, iDuality iin ifuzzy inumber ilinear iprogramming iby iuse iof ia icertain ilinear iranking ifunction, iAppl.iMath.iComp.i180 i(2006) i206-216.

[7]iN.iSafaei, iA inew imethod ifor isolving ifully ifuzzy ilinear ifractional iprogramming iwith ia itriangular ifuzzy inumbers, i3 i(2014) i273-281

[8] iS.iDas iand iT.iMandal, iA isingle istage isingle iconstraints ilinear ifractional iprogramming iproblem: iAn iapproach,Opera.iRese.iand iAppl.: iAn iInter.iJ.i2 i(2015) i1-5.

[9] iS.iM. iHashemi, iM.iModarres, iE.iNasrabadi, iM.iM. iNasrabadi, iFully ifuzzified ilinear iprogramming, isolution iand iduality, iJ.iIntell.iFuzzy iSyst.i17 i(2006) i253-261.

[10] iS.iDas, iT.iMandal, iS.A.iEdalatpanah, iA inew iapproach ifor isolving ifully ifuzzy ilinear ifractional iprogramming iproblems iusing imulti iobjective ilinear iprogramming iproblem, iRAIRO- iOperations iResearch, i51 i(2017)285-297. 
i[11] iS.iDas, iT.iMandal, iS.A.iEdalatpanah,A imathematical imodel ifor isolving ifully ifuzzy ilinear iprogramming iproblem iwith itrapezoidal ifuzzy inumbers, iApplied iIntelligence, i46(2016)509-519.

[12] iS.iDas, iT.iMandal, iS.iA.iEdalatpanah, iA iNew iProcedure ifor iSolving iFuzzy iLinear iFractional iProgramming iProblem: iNumerical iPoint iof iView, iJournal iof iComputational iScience, i(2018) i(In ipress).

[13] iS.iDas, iT.iMandal, iS.iA.iEdalatpanah, i“A inew imethod ifor isolving ifuzzy ilinear ifractional iprograms iwith iTriangular iFuzzy inumbers, iInternational iJournal iof iMathematical iEngineering iand iScience i(IJMES), i4(2015)22-29.

[14] iS.iDas, iT.iMandal, iS.iA.iEdalatpanah, iA inote ion i“ iA inew imethod ifor isolving ifully ifuzzy ilinear ifractional iprogramming iwith ia itriangular ifuzzy inumbers", iApp.iMath.iand iComp.iIntel., i4 i i(2015) i361-367.

[15] iS.iDas, iT.iMandal, iS.iA.iEdalatpanah, iA inew imethod ifor isolving ilinear ifractional iprogramming iproblem iwith iabsolute ivalue ifunctions, iInternational iJournal iof iOperation iResearch, i(2016) i(In iPress).

[16]S.iDas, iT.iMandal, iA igeneral iform iof ifuzzy ilinear ifractional iprograms iwith trapezoidal ifuzzy inumbers, iInternational iJournal iof iData iEnvelopment iAnalysis iand i*Operations iResearch*, i i i2 i(2016), i16-19.

[17] iS.iDas, iT.iMandal, iS.iA.iEdalatpanah, iErratum ito: iA imathematical imodel ifor isolving ifully ifuzzy ilinear iprogramming iproblem iwith itrapezoidal ifuzzy inumbers, iApplied iIntelligence,46(2017) i520-520.

[18] iS.iDas, iT.iMandal, iA inew imodel ifor isolving ifuzzy ifuzzy ilinear ifractional iprogramming iproblem iwith iranking ifunction.iJournal iof iApplied iResearch ion iIndustrial iEngineering.4(2017), i89-96.

[19] iS.iDas, iT.iMandal, iA iMOLFP imethod ifor isolving ilinear ifractional iprogramming iunder ifuzzy i ienvironment, iInternational iJournal iof iResearch iin iIndustrial iEngineering, i6(2017), i202-213.

[20]iS.Das, iModified iMethod ifor iSolving iFully iFuzzy iLinear iProgramming iProblem iwith iTriangular iFuzzy iNumbers, iInternational iJournal iof iResearch iin iIndustrial iEngineering, i6(2017), i293-311.

[21]i iS.iSchaible, iFractional iprogramming iI:duality, iManage.Sci., i22 i(1976) i658-667. 
[22]iVeeramani, iC.iand iM.iSumathi, iSolving ithe ilinear ifractional iprogramming iproblem iin ia ifuzzy ienvironment: iNumerical iapproach.iApplied iMathematical iModelling, i2016.i40(11-12): ip.i6148-6164.

[23] iStanojević, iB. iand i iStanojevic, iM. i, iSolving imethod ifor ilinear ifractional ioptimization iproblem iwith ifuzzy icoefficients in ithe iobjective ifunction, iInternational iJournal iof iComputers iCommunication i\& iControl, i2013, i8:p.136-145.

[24] iSaberi iNajafi, iH. iand iS. iEdalatpanah, iA iNote ion i"A inew imethod ifor isolving ifully ifuzzy ilinear iprogramming iproblems". iApplied iMathematical iModelling, i2013. i37(14): ip. i7865-7867.

[25]Saberi iNajafi, iH., iS.A. iEdalatpanah, iand iH. iDutta, iA inonlinear imodel ifor ifully ifuzzy ilinear iprogramming iwith ifully iunrestricted ivariables iand iparameters. iAlexandria iEngineering iJournal, i2016.

[26]iA.iMehra, iS.iChandra iand iC. iR.iBector, iAcceptable ioptimality iin ilinear ifractional iprogramming iwith ifuzzy icoefficients, iFuzzy iOptimization iDecision iMaking, i6,5$16 \mathrm{i}(2007)$.

[27]iSmarandache, iF.iA iUnifying iField iin iLogics: iNeutrosophic iLogic.iNeutrosophy, Neutrosophic iSet, iNeutrosophic iProbability: iNeutrosophic iLogic.iNeutrosophy, i NeutrosophicSet, iNeutrosophic iProbability, iInfinite iStudy.

[28]iM.iAbdel-basset, iM.iMohamed iand iF.iSmarandache, iLinear ifractional iprogramming ibased ion itriangular ineutrosophic inumbers, international iJournal iof iApplied iManagement i Sciences, i11, i1-20,i(2019).

[29]iP.iBiswas, iS.iPramanik i\& iB.C.iGiri, iTOPSIS imethod ifor imulti-attribute igroup idecesion making iunder isingle-valued ineutrosophic ienvironment, iNeural icomputing iand i Applications,i27(3),727-737.

[30]iM.iDarehmiraki, iA isolution ifor ithe ineutrosophic ilinear iprogramming iproblem iwith ia inew iranking ifunction, iOptimization itheory ibased ion iNeutrosophic iand iPlithogenic iSets, i (2020).

[31]iA.iNafei iet ial.i, iA inew imethod ifor isolving iinterval ineutrosophic ilinear iprogramming ii i i iproblems,ActaPolytechnicaHungarica, i i(2020), i1-17 
[32]iEdalatpanah, iS. iA., i\& iSmarandache, iF.i(2019).iData iEnvelopment Analysis ifor iSimplified iNeutrosophic iSets.iNeutrosophic iSets iand Systems, i29, i215-226.

[33]iEdalatpanah, iS.iA.i(2019).iA iNonlinear iApproach ifor iNeutrosophic Linear iProgramming.iJournal iof iApplied iResearch ion iIndustrial Engineering, i6(4), i358-366.

[34]Edalatpanah, iS.iA.i(2020).A iDirect iModel ifor iTriangular Neutrosophic iLinear iProgramming.iInternational iJournal iof iNeutrosophic Science i, i1(1), i15-24.

[35]Ammar, iE. iS., i\& iEljerbi, iT.i(2019).iOn isolving ifuzzy irough multiobjective iinteger ilinear ifractional iprogramming iproblem.iJournal of iIntelligent i\& iFuzzy iSystems, i(Preprint), i1-13.

[36]Stanojevic, iB., iDzitac, iS., i\& iDzitac, iI.i(2020).iCrisp-linear-and Models iin iFuzzy iMultiple iObjective iLinear iFractional iProgramming. International iJournal iof iComputers, iCommunications i\& iControl,i15(1).

[37]Arya,iR., i\& iSingh, iP.i(2019).iFuzzy iefficient iiterative imethod ifor multi-objective ilinear ifractional iprogramming iproblems.iMathematics and iComputers iin iSimulation, i160, i39-54.

[38]Arya, iR., iSingh,iP., iKumari, iS., i\& iObaidat, iM.iS.i(2019).iAn approach ifor isolving ifully ifuzzy imulti-objective ilinear ifractional optimization iproblems.iSoft iComputing, i1-15.

[39]Nayak, iS., i\& iOjha,iA.iK.i(2019).iMulti-objective iLinear iFractional Programming iProblem iwith iFuzzy iParameters.in iSoft iComputing ifor Problem iSolving i(pp. i79-90).iSpringer, iSingapore.

[40]Nayak, iS., i\& iOjha,iA.i(2019).iOn imulti-level imulti-objective ilinear fractional iprogramming iproblem iwith interval iparameters. RAIRO-Operations iResearch,i53(5), i1601-1616.

[41]Perić, iT., iBabić,iZ., i\& iRešić, iS.i(2020).iA iFuzzy iLinear iProgramming Approach ito iSolve iBi-level iMulti-objective iLinear iProgramming Problems.iIn iAdvances in iOperational iResearch in ithe iBalkans i(pp. 125-135).i 
[42]Mohamed,iM., iAbdel-Basset, iM., iZaied, iA.iN.iH., i\& iSmarandache, iF. (2017). iNeutrosophic iinteger iprogramming iproblem.iInfinite iStudy.

[43]Abdel-Baset,iM., iHezam, iI.iM., i\& iSmarandache, iF.i(2016). Neutrosophic igoal iprogramming.iNeutrosophic iSets i\& iSystems, i11, i25-34.

[44]Mohamed,iM., iAbdel-Baset, iM., iHussein, iN., i\& iSmarandache, iF. Neutrosophic ilinear ifractional iprogramming iproblem.iOctogon Mathematical iMagazine, ivol,25, ino i1, ipp i202-220.i(2017)

[45]Pramanik, iS., i\& iDey, iP.iP.i(2018).iBi-level ilinear iprogramming problem iwith ineutrosophic inumbers.iNeutrosophic iSets iand iSystems, 21(1), i12.

[46]Pramanik, iS., i\& iBanerjee, iD.i(2018).iNeutrosophic inumber igoal programming ifor imulti-objective ilinear iprogramming iproblem iin neutrosophic inumber ienvironment.iInfinite iStudy.

[47]Hezam, iI.iM., iAbdel-Baset, iM., i\& iSmarandache, iF.i(2017). Neutrosophic igoal iprogramming.iInfinite iStudy.

[48]Ahmad,iF., iAdhami, iA.iY., i\& iSmarandache, iF.i(2018).iSingle ivalued neutrosophic ihesitant ifuzzy icomputational ialgorithm ifor imultiobjective nonlinear ioptimization iproblem.iInfinite iStudy.

[49]Smarandache,iF., iAbdel-Basset, iM., i\& iZhou, iY.i(Eds.).i(2017). Neutrosophic iOperational iResearch, iVol. iI.iInfinite iStudy.

[50]Maiti,iI., iMandal, iT., i\& iPramanik, iS.i(2019).iNeutrosophic igoal programming istrategy ifor imulti-level imulti-objective ilinear programming iproblem.iJournal iof iAmbient iIntelligence iand iHumanized Computing, i1-12.

[51]Pramanik, iS., i\& iDey, iP.iP.i(2019).iMulti-level ilinear iprogramming problem iwith ineutrosophic inumbers: iA igoal iprogramming istrategy. Neutrosophic iSets i\& iSystems, i29.

[52]Pramanik, iS., iMallick,iR., i\& iDasgupta, iA.i(2018).iNeutrosophic iGoal Geometric iProgramming iProblem ibased ion iGeometric iMean iMethod iand iits Application.iNeutrosophic iSets iand iSystems, i20(1), i11. 
[53] S.Das, J.K. Dash, (2020). Modified solution for neutrosophic linear fractional programming problems with mixed constraints. International Journal of Research in Industrial Engineering 9(1), 13-24.

[54] S.Das, S.A. Edalatpanah, J.K. Dash (2021). A novel lexicographical based method for trapezoidal neutrosophic linear programming problem, Neutrosophic Sets and Systems, 46(1), 12-26.

[55] J.iYe, iNeutrosophic inumber ilinear iprogramming imethod iand iits iapplication iunder ineutrosophic inumber ienvironments, iSoft iComputing, i22(14)(2018) i4639-4646.

[55]iJ.Ye, iPriortized iaggregation ioperators iof itrapezoidal iintuitionistic ifuzzy isets iand itheir iapplication ito imulticriteria idecision imaking, iNeural iComputation iApplication, i25 i(6) i(2014) i1447-1454. 